This document is the Accepted Manuscript version of a Published Work that appeared in final form in Physical Chemistry Chemical Physics 20, 28741 (2018), copyright CThe Royal Society of Chemistry after peer review and technical editing by the publisher. To access the final edited and published work see

https://pubs.rsc.org/en/content/articlepdf/2018/cp/c8cp05052j 


\title{
Ab Initio Molecular Dynamics Study of Solvated Electrons in Methanol Clusters
}

\author{
Letif Mones, ${ }^{a}$ Gábor Pohl ${ }^{b}$ and László Turi ${ }^{c, 1}$ \\ ${ }^{a}$ Mathematics Institute, University of Warwick, Zeeman Building, Coventry, CV4 7AL, \\ United Kingdom \\ ${ }^{\text {b}}$ Department of Chemistry, Hunter College, CUNY, 695 Park Avenue, New York, NY 10065, \\ USA \\ ${ }^{\mathrm{c}}$ Eötvös Loránd University, Department of Physical Chemistry, Budapest 112, P. O. Box 32, \\ H-1518, Hungary
}

We performed a series of ab initio molecular dynamics simulations to investigate the physical properties of small methanol cluster anions, $\left[\left(\mathrm{CH}_{3} \mathrm{OH}\right)_{\mathrm{n}}\right]^{-},(n=8-32)$. The excess electron was attached to neutral clusters that were prepared to accommodate the electron in interior cavity states or surface bound states. The computed initial binding energies of the electrons to these clusters indicate appealing similarity to the experimentally observed vertical detachment energies. The tendency of the interior state clusters parallels that of the clusters with strong electron binding in the experiments, while the simulated unrelaxed surface state anions are similar to the observed weakly bound species. This assignment is consistent with a previous identification based on hybrid quantum-classical simulations. The time evolution of the cluster anions suggest that interior state electrons slowly move to and relax on the surface, in excess electronic states that appear significantly more stable than the experimentally assigned putative surface states. Based on this result we predict the existence of relaxed surface state isomers of small methanol cluster anions. Due to the kinetic metastability of the experimentally found weakly bound species, we anticipate a serious technical challenge to prepare and identify small methanol cluster anions with relaxed surface states. These more strongly binding surface states

\footnotetext{
${ }^{1}$ Corresponding author. E-mail: turilaszlo@caesar.elte.hu, fax: (36)-1-372-2592
} 
are stabilized by dangling hydroxyl hydrogen atoms pointing to the excess electron's charge distribution. In addition, methyl hydrogens also appear to contribute to the stability of these states. During its transition to the surface, the interior excess electron maintains its initial solvent cavity. No signs of non-cavity interior states are observed in the present first principle ab initio molecular dynamics simulations. 


\section{Introduction}

Solvated electrons are ubiquitous species playing role in a wide range of phenomena of practical and theoretical importance. Besides the hydrated electron, ${ }^{1}$ solvated electrons in alcohols, and in particular, methanol have also been investigated intensively. ${ }^{2-12}$ An almost trivial motivation for studying electron solvation in methanol is to analyze the differences and similarities of electron solvation in water and in "methylated water". A clear experimental manifestation of the replacement of a hydrogen atom to a methyl group is the significantly slower electron solvation dynamics in the bulk solvent. ${ }^{5,6}$ One reasonably expects that the molecular level factors behind the differences in solvation dynamics are easier to identify in a finite size solvent environment, clusters. The presence of the cluster interface, however, adds an additional source of complexity to the problem. ${ }^{13}$ The interfacial phenomenon coupled with the variations in the experimental cluster preparation conditions leads to the appearance of clearly distinguishable trends of the measured vertical detachment energy (VDE) of the excess electron with cluster size. ${ }^{13}$ These patterns are commonly interpreted as signs of the presence of different cluster isomer classes with distinct electron binding motifs. At least three such patterns were observed in water cluster anions. ${ }^{14-16}$ Although several (both experimental ${ }^{15-19}$ and theoretical ${ }^{20-23}$ ) studies have attempted to identify water cluster anion isomers, the debate about the structure, the formation and the relaxation of hydrated electron clusters has not been unequivocally concluded.

The methanol case appears less complicated. The Neumark group found only two markedly different sequences in the excess electron VDE in clusters. ${ }^{10,11}$ Of the two patterns, the higher VDE features (type I) were assumed to indicate structures that solvate the excess electrons in analogy to the bulk solvated electron (interior-bound excess electron states). The more weakly bound structures (type II) were tentatively assigned to the presence of excess electrons that are stabilized at the liquid surface (surface-bound excess electrons). Clearly, the 
experimental conditions of the cluster preparation strongly influence the experimental signals. The main experimental variable, the backing pressure of the supersonic expansion determines the kinetic energy (temperature) of the clusters. Low backing pressure produces warmer, and more likely structurally relaxed clusters, while at high pressure colder, metastable clusters are assumed to dominate. ${ }^{15}$ Neumark and his coworkers report that at low backing pressure the assumed interior-bound state (type I clusters) appears only for $n \sim 140$ and larger, while the putative surface-bound state is not at all evident for $n>190 .{ }^{10}$ Thus, one may hypothesize a transition of the cluster motif in relaxed "warm" clusters with increasing $n$ occurring in the approximate range $140<n<190$. At high backing pressure, in "cold" clusters, the relatively weakly bound surface state appears for all $n>70$, while the interior-bound state persists only for $n>140$.

In the last two decades, significant efforts have been made to understand the molecular details underlying electron solvation in bulk methanol and methanol clusters using computer simulations. One-electron mixed quantum-classical molecular dynamics (QCMD) that treat the excess electron quantum mechanically, while the solvent bath is described classically, focused on reproducing the physical properties of solvated electrons in bulk methanol. ${ }^{24-30}$ Despite the good qualitative agreement with experimental data, the persisting inaccuracies (i.e. the peak position and the shape of the simulated absorption spectrum of the solvated electron $)^{25}$ motivated the improvement of the electron-methanol molecule interaction model using a static exchange pseudopotential theory. ${ }^{31}$ The simulations applying the new potential ${ }^{31}$ notably improved the position and the half-width of the optical spectrum of the solvated electron in bulk methanol in comparison with experiments. ${ }^{4}$ The computed structural features were also predicted in excellent agreement with electron spin echo signals. ${ }^{3}$ More recently, we mimicked low energy electron attachment to pre-existing methanol clusters by investigating excess electron localization on neutral equilibrated methanol clusters. ${ }^{32}$ The observation that the pre- 
existing instantaneous dipole moment of the neutral clusters can bind the excess electron is similar to the aqueous case. ${ }^{33}$ Due to the fact that the surface of the neutral methanol clusters is covered predominantly by the non-polar methyl groups, as suggested by earlier experiments, ${ }^{34}$ initially the electron binds very weakly to the cluster surface. The latest simulation study predicts two main persisting localization modes of the excess electron in equilibrated methanol clusters at $\sim 200 \mathrm{~K},{ }^{35}$ in an apparent agreement with photo-electron imaging experiments. ${ }^{10,11}$ The two localization patterns have strikingly different physical properties, and appear in comparable cluster sizes to those observed. Smaller clusters $(n \leq 100)$ tend to localize the electron in weakly bound, diffuse electronic states on the surface of the cluster. These surface state cluster anions are significantly more weakly bound than the surface states of the anionic water clusters due to the lack of stabilizing free hydroxyl groups on the relaxed methanol cluster surfaces. Larger methanol clusters $(n \geq 200)$ stabilize the electron in compact solvent cavities, in interior-bound states. The interior states exhibit properties that largely resemble and smoothly extrapolate to those simulated for the solvated electron in the bulk. ${ }^{35}$ One has to note, however, that although these QCMD simulations offer a rational alternative to the more demanding fully quantum dynamical simulations, the simulation results can be interpreted at most as semi-quantitative. The limitations of the simulation technique are mostly due to the application of the electron-methanol molecule pseudopotential and the non-polarizable classical interaction model. ${ }^{31}$

While several many-electron quantum molecular dynamics studies have been performed for excess electrons in bulk water and water clusters, ${ }^{23,36-42}$ similar investigations for the solvated electron in methanol have not been carried out so far. The aqueous ab initio MD studies, however, shed light on important general aspects of solvated electron simulations. Allelectron quantum simulations for hydrated electrons are still severely limited by the computational resources. In this context, both the applied computational method (including the 
basis set), the size of the investigated system, and the length of the dynamical trajectories must be carefully selected and optimized. Of the electronic structure methods, the most reasonable choice has been the use of density functional theory (DFT) based methods with large, diffuse basis sets. ${ }^{40,41}$ Within the DFT framework, the selection of the optimal functional represents a further challenge, as illustrated by hydrated electron ab initio molecular dynamics simulations ${ }^{23,36-41}$ carried out by applying either the $\mathrm{PBE}^{43,44}$ or the BLYP functionals. ${ }^{45,46}$ Correlated ab initio calculations on water and methanol cluster anions, however, demonstrated that proper benchmarking of the applied functionals and basis sets is of essential importance if one aims to go beyond the semi-quantitative interpretation of the experimental data. ${ }^{47,48} \mathrm{We}$ found that while neither the PBE, nor the BLYP functionals reproduce the VDE of water and methanol cluster anions with sufficient accuracy, the long range corrected BLYP (LCBLYP) $)^{45,46,49}$ and the hybrid BHandHLYP functional ${ }^{50,51}$ provide a much better estimate. The available hardware also places a limit on the size of the investigated system and the length of the trajectories. The most extensive hydrated electron simulations included 105 water molecules with fully quantum mechanically treated electronic wavefunction propagated along trajectories of $<10$ ps long. ${ }^{23}$ These observations are instrumental in selecting the most suitable computational model for solvated electron simulations in methanol clusters, as we illustrate below.

Despite the relative simplicity of the assignment of methanol cluster anion isomers compared to the aqueous case, there are also important questions that need clarification. The aim of the present paper is to understand the microscopic aspects of electron solvation in small methanol clusters using ab initio molecular dynamics simulation techniques. This study thus necessarily goes beyond the previously used simple one-electron model. One of the most basic questions that cannot be truly addressed in the one-electron picture concerns the structure of the solvated electron, namely, whether the excess electron is stable in a solvent void (cavity 
model), or, alternatively, it prefers a more diffuse distribution, delocalizing on several solvent molecules, similar to that proposed for water not long ago. ${ }^{52}$ In clusters, the position of the electron's localization site relative to the cluster framework (i.e. interior vs. surface excess electron states) may also be a matter of scientific interest. What are the preferred stabilization modes of the electron in the clusters, and what molecular configurations support the specific binding modes? It may also be useful to predict the size of the methanol clusters that are able to bind the excess electron in a certain binding mode, and relate the theoretical estimates of the critical size to the experimental findings. Furthermore, it is also our goal to compare the ab initio MD predictions to previous QCMD results, and to test, retrospectively, the applicability of the one-electron QCMD approach.

\section{Methods}

To investigate the physical properties of methanol cluster anions, we performed ab initio molecular dynamics (AIMD) simulations on three different size $\left(\mathrm{CH}_{3} \mathrm{OH}\right)_{\mathrm{n}}^{-}$anions consisting of $n=8,16$ and 32 molecules. The initial cluster configurations were taken from trajectories of our previous QCMD simulations. ${ }^{35,48}$ In these studies, one-electron simulations were used to generate cluster configurations of different $\operatorname{size}^{35}$ that, in turn, were analyzed in many-electron computations. ${ }^{48}$ In the QCMD part, we produced two 100 ps long equilibrium trajectories of canonical ensembles at $200 \mathrm{~K}$, one for $\left(\mathrm{CH}_{3} \mathrm{OH}\right)_{128}^{-}$clusters with interior state excess electrons, and one for $\left(\mathrm{CH}_{3} \mathrm{OH}\right)_{50}^{-}$clusters with surface-bound excess electrons. ${ }^{35}$ Here, we select ten configurations from each of these equilibrium trajectories, evenly separated by $10 \mathrm{ps}$ time segments. Of the QCMD generated classical configurations, we create smaller clusters of various size that serve as the initial configurations for the ab initio molecular dynamics runs. The small clusters are generated similarly to those in our previous studies. ${ }^{47,48} \mathrm{We}$ determine the center of mass of the solvated electron using the one-electron pseudopotential, and cut out 
clusters of gradually increasing size consisting of $n$ methanol molecules $(n=8,16$, and 32) that are nearest to the center of mass of the electron. These clusters are thought to model the first, the second and (partly) the third solvation shells of the excess electron. We note that we distinguish two types of structures, interior and surface state structures. These names, however, refer only to the electron binding motifs of the QCMD simulated parent clusters. In the interior configurations the methanol molecules surround a cavity with their $\mathrm{OH}$ bonds pointing toward the cavity. This arrangement is similar to that found in the interior state structure of water cluster anions. ${ }^{47}$ In surface state configurations the dipole moment of the cluster and possibly dangling OH hydrogens localize the excess electron. ${ }^{48}$

In the present work we use DFT for the electronic structure calculations with the BHandHLYP functional. ${ }^{50,51} \mathrm{We}$ have recently shown $^{48}$ that due to its Hartee-Fock (HF) exchange component this functional with the diffuse $6-31(1+3+) G(d)$ basis set provides a reasonable estimate of the VDE values of small methanol clusters anions $(n=2-8)$. We also demonstrated that the BHandHLYP VDE values strongly correlate with those of MP2 using the same basis set. ${ }^{48}$ The use of the MP2/6-31(1+3+)G(d) model in comparisons here and later in the text can be justified by our earlier finding that reveals a strong correlation between the VDE values computed at MP2/6-31(1+3+)G(d) and eom-EA-CCSD/aug3-cc-pVDZ levels for small water cluster anions. ${ }^{47}$

Simulations were performed using the $\mathrm{CP} 2 \mathrm{~K}$ program package, ${ }^{53}$ which applies the Gaussian and plane wave method (GPW) in combination with the Goedecker-Teter-Hutter pseudopotentials. ${ }^{54,55}$ The GPW method uses a dual basis set representation (contracted Gaussian functions and auxiliary plane waves) of the electron density to efficiently solve the corresponding Kohn-Sham equations. For the localized basis set our starting point is the MOLOPT-TZVP basis, which is optimized for molecular systems in both gas and condensed phases. ${ }^{56}$ We augmented this basis set by the diffuse functions of the $6-31(1+3+) \mathrm{G}(\mathrm{d})$ basis ${ }^{57}$ 
that was created by adding two additional diffuse $s$ orbitals on the hydrogen atoms to the 6$31++\mathrm{G}(\mathrm{d})$ basis set. ${ }^{58}$ We denote this basis set by aug-MOLOPT-TZVP. The most expensive part of the model is apparently the computation of the HF exchange energy. To reduce the corresponding computational cost we used the Auxiliary Density Matrix Methods (ADMM) ${ }^{59}$ with the diffuse aug-FIT3 basis set. For the plane wave basis set we used an energy cutoff of 480 Ry. For each system a cubic simulation cell was used with a box size of $25 \AA$. For treating long range interactions we applied the Martyna-Tuckerman Poisson solver. ${ }^{60}$ Molecular dynamics simulations in the canonical (NVT) ensemble were carried out using 1.0 fs timestep at $200 \mathrm{~K}$. The temperature was regulated by the Canonical Sampling through Velocity Rescaling (CSVR) thermostat ${ }^{61}$ using a time constant of 100 fs. To compute VDE, we also performed single point calculations on selected configurations along the MD trajectories without the excess electron, using the same computational method as for the anions. For comparison we also performed VDE calculations using the Gaussian09 program package ${ }^{62}$ using the MP2 and BHandHLYP methods with the atom centered 6-31(1+3+)G(d) basis set. The molecular structures and spin densities were created and analyzed using the GaussView program of the Gaussian09 program package.

\section{Results and Discussion}

Preformed interior state clusters. We start the discussion with the clusters that have preformed interior excess electronic states. The motivation to launch the dynamics from preformed interior states is (at least) threefold. a) The interpretation of photo-electron imaging experiments ${ }^{10,11}$ predicts interior electron binding motifs in methanol cluster anions, analogous to the interior states of water cluster anions. One may wonder whether such states persists in small methanol clusters. b) A strongly related interesting problem concerns the critical size of the transition of the excess electron's localization mode with increasing cluster size, from the 
surface to the interior (or vice versa). c) The temporal evolution of the cavity after injecting an electron in the interior of the cluster may also serve important information on the structure of the solvated electron.

Representative clusters with preformed interior states are shown for $n=8,16$ and 32 sizes in Figure 1 with the computed spin density isosurfaces. The spin density isovalues are chosen to be 0.0004 throughout the paper, unless noted otherwise. These clusters were carved out from the same $n=128$ interior state cluster configuration of our previous QCMD simulations. ${ }^{35}$ Figure 1 illustrates that the initial cavity clusters considered in this work support localized interior state excess electron distribution. It is, however, clear that for the smallest clusters $(n=8)$ the spin density noticeably spreads out beyond the first solvation layer. The presence of the second and third layers seem to be sufficient to contain the electron within the preformed cavity. The calculated VDE values indicate strong excess electron binding for these clusters. The present model of the ab initio MD simulations (BHandHLYP/GPW+augMOLOPT-TZVP) predicts VDE values in the 440-990 meV range with an average of $670 \mathrm{meV}$ for the selected 10 configurations of the $n=8$ clusters. We found a very good agreement between these VDE values and those computed in our previous ab initio benchmark study using the MP2/6-31 $(1+3+) \mathrm{G}(\mathrm{d})$ model $^{48}$ with a slight and systematic, $\sim 40 \mathrm{meV}$ average overestimation. A more extensive comparison can be found in Figure S1 of the Supplementary Information (SI) showing a correlation diagram including VDE values for 100 configurations of the $n=8$ clusters. Increasing the cluster size from $n=8$ to 16 increases the VDE in every single case to the $1070-1930 \mathrm{meV}$ range. The second layer of molecules clearly further stabilizes the excess electron. This systematic tendency ends when one adds the next solvation sphere to the cluster. Of the 10 initial configurations, we find $40 \%$ where the addition of 16 extra molecules (as a third solvation layer) to the $n=16$ clusters results in less strongly bound excess electrons. In one case, for example, we compute 697, 1215 and $1100 \mathrm{meV}$ VDE for the 
corresponding $n=8,16$ and 32 clusters, respectively. Details are shown in Table S1 of the Supplementary Information (SI). Addition of 8 molecules to the $n=8$ cluster increases the VDE by $400-900 \mathrm{meV}$, an increase of 70-200\% relative to the $n=8$ VDE. Going from $n=16$ to 32 changes the VDE by $-200-400 \mathrm{meV}$, or $-10-30 \%$ of the $n=16 \mathrm{VDE}$. It is clear that irrespective of the fact whether the VDE increases or decreases by the addition of the third layer, the presence and the configuration of approximately three solvation layers (32 molecules) strongly influence the stability of the excess electron, although the third layer significantly more weakly than the first two.

Next we discuss the dynamics of methanol cluster anions with preformed internal states by following the temporal evolution of the vertical detachment energy of the excess electron. For the smallest investigated cluster size $(n=8)$, the molecular frame tends to break up to smaller hydrogen-bonded units, while the preformed interior electron binding site quickly disintegrates. The time evolution of the VDE for the one MD trajectory that was simulated for the extended 10 ps period, is shown in Figure 2. Here we observe that the cavity structure is preserved for about 1 ps with VDE in the $400-900 \mathrm{meV}$ range, followed by a quick decrease to $\sim 0 \mathrm{meV}$ within the next 1 ps (see also Figure S2 of SI). Our observations for this longer trajectory match the general VDE trends for the other 9 trajectories, suggesting that methanol cluster anions of this size are likely to be unstable at the simulated $T=200 \mathrm{~K}$ (Figure S3 of SI). Once again, we emphasize that due to the finite basis set and the finite dimensions of the simulation box the particular dynamical details of this scenario following a hypothetical excess electron localization in a preformed solvent cavity convey no physical information beyond the fact that the VDE becomes essentially zero. This clearly indicates that the electron has a tendency to spontaneously detach from the cluster.

The dynamics of the preformed cavity clusters containing $n=16$ molecules do not exhibit significant relaxation within the first ps (Figure S4 of SI). The excess electron density 
remains mostly within the clusters, but in half the cases it moves at least partially to the surface by 1 ps. Inspection of the 10 ps long trajectories (of which we simulated two) reveals that the tendency to move to the surface continues. This is illustrated in Figure 3 on four snapshots of a selected trajectory at $t=0 \mathrm{ps}, 1 \mathrm{ps}, 4 \mathrm{ps}$ and $10 \mathrm{ps}$. We visually estimate that the process is completed by $\sim 5$ ps. The time evolution of the VDE shows that the VDE of the surface electron in the $t=5-10$ ps period fluctuates around an average value of $\sim 800 \mathrm{meV}$ (Figure 4). For the other long trajectory the electron stays in the cavity for a longer period up to $\sim 7$ ps with a VDE plateau at $\sim 1.4 \mathrm{eV}$ (Figure S5 of SI). After 7 ps of dynamics, the electron moves to the surface with a concomitant decrease of VDE to $\sim 600 \mathrm{meV}$ by 10 ps. Clearly, for this trajectory the excess electron has a significant surface character at $t=10 \mathrm{ps}$, but it is still partially embedded in the interior of the cluster (Figure S5 of SI). For both trajectories we observe that the transition of an interior state electron to a surface state electron is a smooth, gradual process. During its diffusion, the excess electron preserves the surrounding solvent cavity with $\mathrm{OH}$ hydrogens pointing toward the electron's charge distribution. Although at the surface the cavity opens, the electron remains partially solvated from the interior with $\mathrm{OH}$ hydrogens. These dangling hydrogens, of which three interacts with the excess electron in the structure at $t=10 \mathrm{ps}$ in Figure 3, appear to be essential in stabilizing these relaxed surface state electrons.

For the $n=32$ cluster we performed two simulations, generating a 1 ps and a 4 ps long trajectories. The shorter trajectory does not reveal major features, its VDE starting at $1.1 \mathrm{eV}$ and relaxing to about $0.8 \mathrm{eV}$ by $1 \mathrm{ps}$ (Figure $\mathrm{S} 6$ of $\mathrm{SI}$ ). Although the excess electron is still located in a cavity, it has clearly moved toward the surface. Similar changes are evident for the longer trajectory. The dynamics here exhibits a more characteristic tendency with the VDE starting at $1.7 \mathrm{eV}$, practically monotonically decreasing to $0.1 \mathrm{eV}$ and jumping back and stabilizing at around $0.8-1.0 \mathrm{eV}$ (Figure 5). Although the transition of the cavity state to the surface state does seem to be fully completed by the end of the trajectory, significant portion of 
the excess electron is still partially embedded in the cluster (Figure 6). It is mostly the dangling $\mathrm{OH}$ hydrogen atoms that stabilize the electron at the surface similarly to the $n=16$ case above. In this particular case, however, one can recognize that methyl hydrogens also appear in the vicinity of the excess electron distribution (Figure 7). This observation is consistent with the findings of an early DFT based quantum chemical study that predicted noticeable contribution of electron - methyl hydrogen interactions to the stabilization of an excess electron in methanol. ${ }^{63}$ Figure 6 also reveals an interesting feature of this particular $n=32$ trajectory. The formation of regular substructures, linear chains is likely to signal a phase transition from a liquid to a solid molecular environment at $200 \mathrm{~K}$.

Preformed surface state clusters. These clusters may be viewed as representing the initial conditions after low kinetic energy electron attachment to neutral cluster. Figure 8 shows a set of representative methanol clusters anions with preformed surface excess electronic states for cluster sizes $n=8,16$ and 32 . These clusters were carved out from the same $n=50$ surface state cluster configuration of our previous QCMD simulations. ${ }^{35}$ The spin densities at $t=0$ illustrate diffuse excess electron distributions at the cluster surface. These states appear to be very weakly bound as was demonstrated for the $n=8$ clusters in our previous benchmark study with most of the VDE values (at the MP2/6-31(1+3+)G(d) level) falling in the $0-100 \mathrm{meV}$ range. ${ }^{48}$ Here we found that our $\mathrm{CP} 2 \mathrm{~K}$ calculations for the same initial configurations result in VDE values that are in average $\sim 140 \mathrm{meV}$ lower than the previous MP2 numbers. For the $n=$ 16 case we observe similar, slightly negative VDE values for the initial clusters. In both cases, the essentially zero (or slightly negative) initial VDEs of the present simulations suggest that the presumably weakly bound, diffuse excess electrons (predicted by the MP2 calculations) are artificially localized somewhat and, thus, destabilized in the vicinity of the cluster by the finite dimensions of the simulation cell. Despite this technical difficulty, we initiated MD simulations from these structures to check whether they are able to relax to more localized electron 
distributions during the dynamics. We anticipate the VDE of these structures to be in better agreement with the MP2 calculations. In fact, for the $n=8$ surface state cluster dynamics we find that with the exception of a single trajectory, the VDE always climbs up to $200 \mathrm{meV}$ or higher, even reaching $\sim 500 \mathrm{meV}$ in a single occasion. However, in most trajectories the stabilization of the excess electron appears only temporary, with the VDE quickly falling back to $\sim 0$ again. The time evolution of the VDE for these trajectories is shown in Figure S7 of SI. All these tendencies imply that the $n=8$ surface state cluster anions are unlikely to be stable with respect to the autodetachment and/or the fragmentation of the cluster. To test the capabilities of the present computation model for more strongly bound surface electrons, we selected ten surface state configurations along the $n=8$ surface state cluster trajectories with VDE values between 150 and $500 \mathrm{meV}$, and computed their VDE's using the MP2/6$31(1+3+) G(d)$ and the BHandHLYP /6-31(1+3+)G(d) models. We found that for the selected configurations the agreement is acceptable (Table S2 of SI), although it is not as satisfactory as for the interior states (Figure S1 of SI). In general, we note that the BHandHLYP/GPW+augMOLOPT-TZVP calculated VDE values for surface state configurations that reasonably bind the excess electron slightly overestimate the MP2/6-31(1+3+)G(d) ones. This trend for the BHandHLYP functional is consistent with our previous findings. ${ }^{48}$

Next, we followed the $n=16$ methanol cluster anion dynamics for ten trajectories. Most of the trajectories predict very low VDE values suggesting general instability of these clusters with respect to excess electron binding (Figure S8 of SI). Interestingly, however, two trajectories significantly stabilize the surface electron within $\sim 0.5 \mathrm{ps.} \mathrm{We} \mathrm{extended} \mathrm{the} \mathrm{length}$ of the simulation for these trajectories to $10 \mathrm{ps}$, and observe that one of these trajectories reaches a stable VDE plateau after 3-4 ps dynamics with the VDE fluctuating around $600 \mathrm{meV}$ (Figure 9). This cluster forms stable surface states (at least, within the timeframe of the simulation) with at least one $\mathrm{OH}$ group pointing toward the excess electron (Figure 9). The VDE of the other 
trajectory suddenly becomes very low after about $4 \mathrm{ps.} \mathrm{This} \mathrm{indicates} \mathrm{that} \mathrm{this} \mathrm{cluster} \mathrm{is} \mathrm{unlikely}$ to bind the excess electron for an extended period, and the electron eventually detaches from the cluster. For the $n=32$ case we simulated two short, 1 ps long trajectories. Here both trajectories started from very diffuse excess electron distributions with slightly negative VDE values. Although these initial configurations are not expected to bind the excess electron, and the electron is constrained to the vicinity of the cluster only by the finite dimensions of the basis set and the simulation cell, we performed simulations to check whether one could find surface state configurations during the course of the dynamics that actually bind the electron. Once one locates such a configuration, it is to be seen if these surface states with high VDEs persist or not. For the two trajectories we found two different scenarios (Figure S9 of SI). One of the cluster anions remains unstable during the dynamics and does not bind the excess electron (Figure S9, bottom), while the other becomes stable after $\sim 0.5$ ps with the VDE jumping from below zero up to $\sim 500 \mathrm{meV}$, then within another $300 \mathrm{fs}$ destabilizes again (Figure S9, top). It then presumably loses the excess electron in a spontaneous autodetachment process. The common feature of the $n=32$ trajectories with preformed surface states is that we were unable to locate persisting surface states.

\section{Conclusions}

We have performed ab initio molecular dynamics simulations to investigate the adiabatic relaxation of negatively charged methanol clusters following electron attachment to neutral clusters. These clusters were prepared in mixed quantum-classical molecular dynamics simulations to represent possible interior and surface state binding motifs for the excess electron. For the interior states QCMD simulations predicted solvent voids in the interior of the clusters as binding sites. We find that these QCMD created pre-formed cavity states strongly bind the excess electron. The dual basis representation of the computational model of the 
present work produces VDE values for interior state anions in good overall agreement with previous ab initio numbers using atom centered basis sets. ${ }^{48}$ For the configurations that support surface bound excess electron states in QCMD simulations we observe very weak excess electron-cluster interaction. In fact, these interactions appear nearly neutral with the computational model of this study predicting slight negative values for the VDE. These values represent $0.1-0.2 \mathrm{eV}$ underestimation relative to previous MP2 values. ${ }^{48}$ Also, we have observed an opposite tendency for the VDE values of more localized, more strongly bound surface states with $0.1-0.2 \mathrm{eV}$ overestimation of the MP2 data. It is also to be noted that the one-electron pseudopotential based VDE values for both the interior and surface state configurations reasonably reproduced the results of many-electron quantum mechanical calculations. $^{48}$

The dynamics of both the interior and surface state anionic clusters reveal that although the initial cavity state anions are significantly stabilized relative to the neutral clusters, and the initial surface state structures bind the excess electron only very weakly, the excess electrons have a definite tendency to move to the surface of the cluster. For the smallest size $(n=8)$ the clusters quickly fragment to smaller clusters, while the VDE approaches zero. This observation is valid for both types of structures. For larger cluster sizes, however, we clearly observe that the excess electron slowly diffuses to the surface, while the cluster structures remain intact. For both the $n=16$ surface and interior state anions, and the $n=32$ interior case we find persisting surface excess electronic states with $0.6-1.0 \mathrm{eV}$ stabilized VDE (see Figures 4, 5 and 9). It is especially interesting to notice that for the $n=16$ size, the electron stabilizes on the surface regardless of the initial position of the excess electron. In general, one also observes that initially the interior states have significantly higher VDE values than the final relaxed surface states. The largest examined $\left(\mathrm{CH}_{3} \mathrm{OH}\right)_{32}^{-}$clusters with preformed cavity states show similar relaxation behavior to the $n=16$ clusters. We note that the final relaxed surface VDE for $n=$ 
32 is somewhat higher, $\sim 1.0 \mathrm{eV}$, in the only studied longer trajectory than for the $n=16$ clusters. The VDE values of the relaxed surfaces states can also be placed in context with the experimental observations. Although cluster anions were not observed in experiments for $n<$ 70, the extrapolation of the experimental tendencies for smaller clusters may be instructive. The simulated $0.6-1.0 \mathrm{eV}$ relaxed surface state VDE values for $n=16$ and 32 are higher (even taking the slight overestimation of the VDE by the employed computational model into account) than the experimentally found $\sim 0.2 \mathrm{eV}$ for the smallest observed type II cluster $(n=$ 70). Also, these values are lower than the VDE values of type I clusters extrapolated to this size $(\sim 1.5 \mathrm{eV}$ for $n=16, \sim 1.9 \mathrm{eV}$ for $n=32)$. Nevertheless, we notice that the initial cavity states of our simulations stabilize the electron by about this same energy, $\sim 1.5 \mathrm{eV}$, suggesting that the experimental VDEs of type I clusters extrapolated to the cluster sizes of the simulation are comparable to those of the simulated cavity states. The correlation between type II clusters and the simulated surface state clusters is less clear. Our simulations suggest that relaxed surface clusters have greater VDE $(0.6-1.0 \mathrm{eV})$ than those expected for the experimental type II clusters at the same cluster size $(\lesssim 0.2 \mathrm{eV})$. This may reasonably suggest that type II clusters are not relaxed surface state clusters, but represent kinetically hindered, low temperature species that bind the excess electron in a metastable state. One can then speculate that the initial electron binding to neutral clusters takes place in these weakly bound (presumably surface) states, an assumption that is in accord with the low VDE values of the initial surface state anions of the present study. At low cluster temperatures (higher backing pressure) these structures (type II) dominate the experiments in the $n=73-458$ size range, but type I clusters also appear in a gradually increasing ratio with increasing cluster size. ${ }^{10}$ However, one finds no experimental indication for the presence of an intermediate type of isomer belonging to the simulated relaxed surface state anions. It is conceivable that the relaxation of the kinetically trapped initial excess electronic states that are localized on the methyl group covered cluster surface, involves the 
formation of free (dangling) hydroxyl hydrogen atoms. This step probably takes relatively long compared to the relaxation of an already partly relaxed surface electron to a cavity electron. If one increases the cluster temperature (decrease the backing pressure in the cluster preparation) type I clusters prevail, ${ }^{10}$ suggesting that the initial surface electrons relatively quickly relax to the interior of the cluster. Note that the hypothesis of the initial electron attachment to the neutral cluster surface implies that the direction of the relaxing electron's displacement is from outside to inside, a tendency we also observed for the relaxation of large methanol cluster anions in QCMD simulations. ${ }^{32,35}$ In the present AIMD study, however, we found that small cluster anions $(n \leq 32)$ relax to configurations that bind the excess electrons in surface states. It appears plausible that a critical size is needed to accommodate the electron in the interior of the cluster. On the other hand, experiments clearly point to the difficulty to prepare cluster anions of this small size. We anticipate that the preparation of small neutral clusters may be feasible only at very low temperatures. Once the ultracold small neutral clusters are formed, however, the electron - neutral cluster interaction that depends on the dipole moment of the cluster, may not be sufficient to attach the excess electron to the cluster. Even if an excess electron binds to these ultracold small methanol clusters, the resulting metastable state will probably persist on the timescale of the experiment, preventing the observation of the relaxed surface state configurations. So, even though we predict that small methanol cluster anions can stabilize the excess electron in relaxed isomer configurations that support surface excess electronic states, the experimental verification of this prediction poses a challenging problem. In particular, we find it conceivable that the predicted relaxed surface state configurations may not be accessible from the metastable initial states of low energy electron attachment experiments to preexisting small methanol clusters.

Structural information is also readily accessible from our AIMD simulations. It is especially instructive to consider the solvent structure around the solvated excess electrons. We 
find that the initially preformed interior states keep their solvent cavities during the timeframe of the dynamics. We do not observe collapse of the cavity electrons to such non-cavity states that were proposed for the structure of the hydrated electron. ${ }^{52}$ Here, we find it important to point out that our AIMD simulations support the cavity solvation structure around the solvated electron in the interior of methanol cluster anions. The spin density distributions of the interior electrons have similar features to those found by Walker and Bartels, ${ }^{64}$ of which we emphasize the presence of the solvent void and apparently some (small) part of the spin density localizing on the nearest oxygen and carbon atoms. Since the present interior state simulations were initiated from preformed cavities, and the interior state electrons tend to move toward the surface of the clusters, it is difficult to infer precisely the degree of localization of the excess electron within and outside the solvent cavities. Our simulations for a typical interior state configuration ( $t=1$ ps structure on Fig. 6 ) indicate that $\sim 70 \%$ of the spin density is concentrated in the cavity, within $\sim 3 \AA$ around the center. This estimate is in good qualitative accord with previous AIMD simulations for the hydrated electron in water, ${ }^{40}$ and in aqueous glucose solutions. ${ }^{65}$

\section{Conflict of Interest}

There are no conflicts to declare.

\section{Acknowledgements}

This work was supported by research grants to L.T. from the National Research, Development and Innovation Office, Hungary (NKFIH, previously OTKA, K104237 and K128136). 


\section{Figure Captions}

Figure 1. Initial methanol cluster anion configurations with preformed cavity states with $n=8$, 16 and 32 molecules. Blue indicates positive spin density, while green shows negative spin density areas. The isosurfaces contain $76 \%, 82 \%$ and $82 \%$ of the spin density, respectively. Theses clusters were carved out of the same $n=104$ interior state methanol cluster anion configuration of a previous QCMD trajectory. ${ }^{35}$

Figure 2. A typical time evolution of the VDE of methanol cluster anions with $n=8$ (see Trajectory \#4 in Figure S3 of SI). The dynamics starts from a preformed cavity state.

Figure 3. Four snapshots of a typical trajectory of methanol cluster anions with $n=16$ molecules at $t=0 \mathrm{ps}, 1 \mathrm{ps}, 4 \mathrm{ps}$ and $10 \mathrm{ps}$. The excess electron starts the dynamics from a preformed cavity state and ends up in a surface state.

Figure 4. A typical time evolution of the VDE of methanol cluster anions with $n=16$ molecules (see Figure 3 and Trajectory \#6 in Figure S4 of SI). The excess electron starts the dynamics from a preformed cavity state and ends up in a surface state.

Figure 5. The time evolution of the VDE of a methanol cluster anion with $n=32$ molecules. The excess electron starts the dynamics from a preformed cavity state and moves toward the surface (see Figure 6). Note that the minimum VDE at $1550 \mathrm{fs}$ corresponds to the third structure from the top in Figure 6.

Figure 6. Four snapshots of a trajectory of methanol cluster anions with $n=32$ molecules at $t$ $=0,1,1.55$ and 4 ps (see Figure 5). The excess electron starts the dynamics from a preformed cavity state and clearly moves toward the surface.

Figure 7. The spin density distribution of a relaxed surface state $\left(\mathrm{CH}_{3} \mathrm{OH}\right)_{32}^{-}$methanol cluster anion. The isosurface contains $71 \%$ of the spin density. The electron localizes on the cluster surface, and is stabilized by dangling hydroxyl hydrogen atoms and also interacts with the methyl groups. The structure is identical to that at the bottom in Figure 6. 
Figure 8. Initial methanol cluster anion configurations with preformed surface states with $n=$ 8, 16 and 32 . The isosurfaces contain $23 \%, 35 \%$ and $30 \%$ of the spin density, respectively. These clusters were carved out of the same $n=50$ surface state methanol cluster anion configuration of a previous QCMD trajectory. ${ }^{35}$ Due to the diffuse electron distribution, the spin density surfaces are shown with isovalue of 0.0002 .

Figure 9. Time evolution of the VDE of methanol cluster anions with $n=16$ molecules for two $10 \mathrm{ps}$ long trajectories. The excess electron starts the dynamics from a preformed surface state and ends up in a surface state. The final structures of the dynamics are also shown. Note that while the top figure shows the electron stabilizing in a persisting surface state (with spin density isovalue 0.0004), the excess electron becomes weakly bound and likely detaches from the cluster in the bottom figure (with spin density isovalue 0.0002). 
Figure 1.

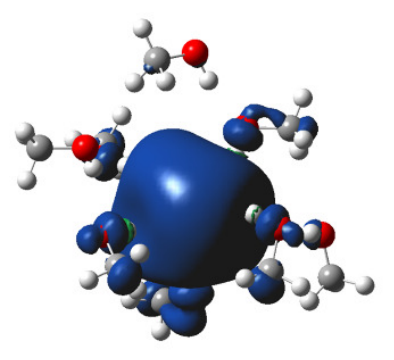

$\left(\mathrm{CH}_{3} \mathrm{OH}\right)_{8}^{-}$

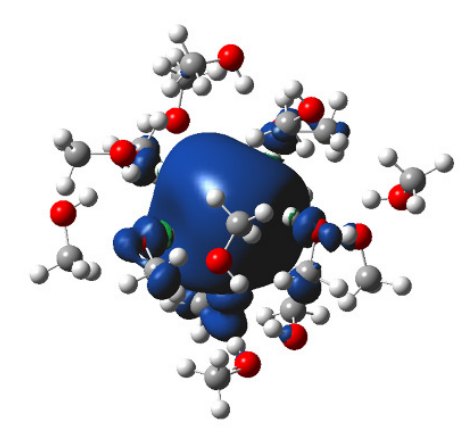

$\left(\mathrm{CH}_{3} \mathrm{OH}\right)_{16}^{-}$

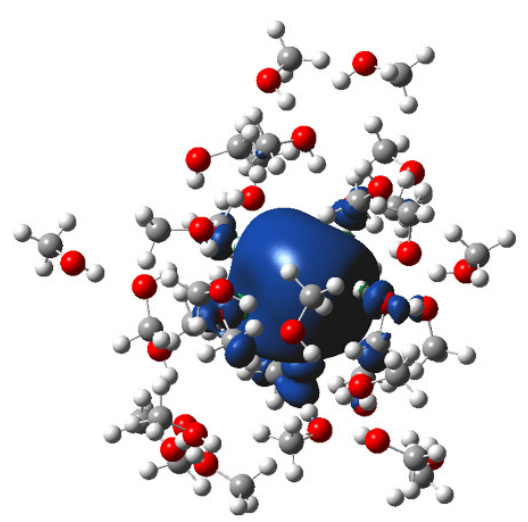

$\left(\mathrm{CH}_{3} \mathrm{OH}\right)_{32}^{-}$ 
Figure 2.

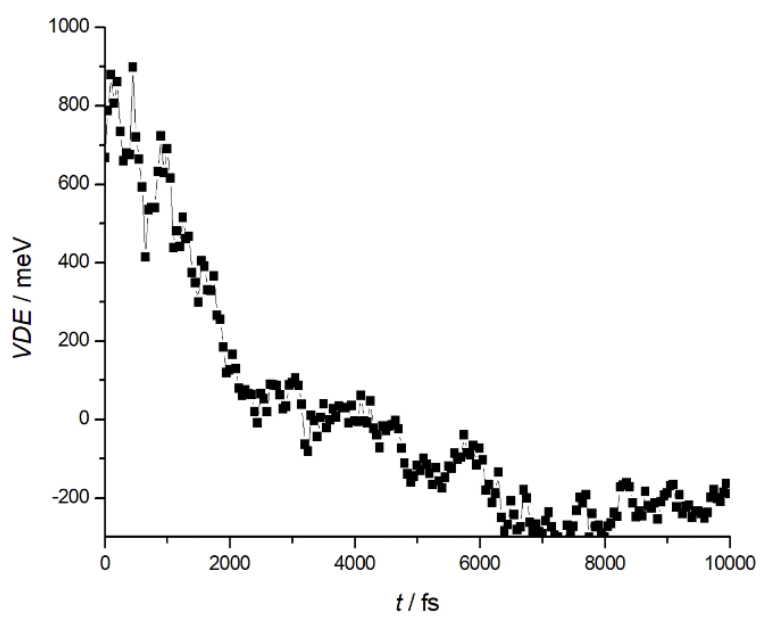

24 
Figure 3.
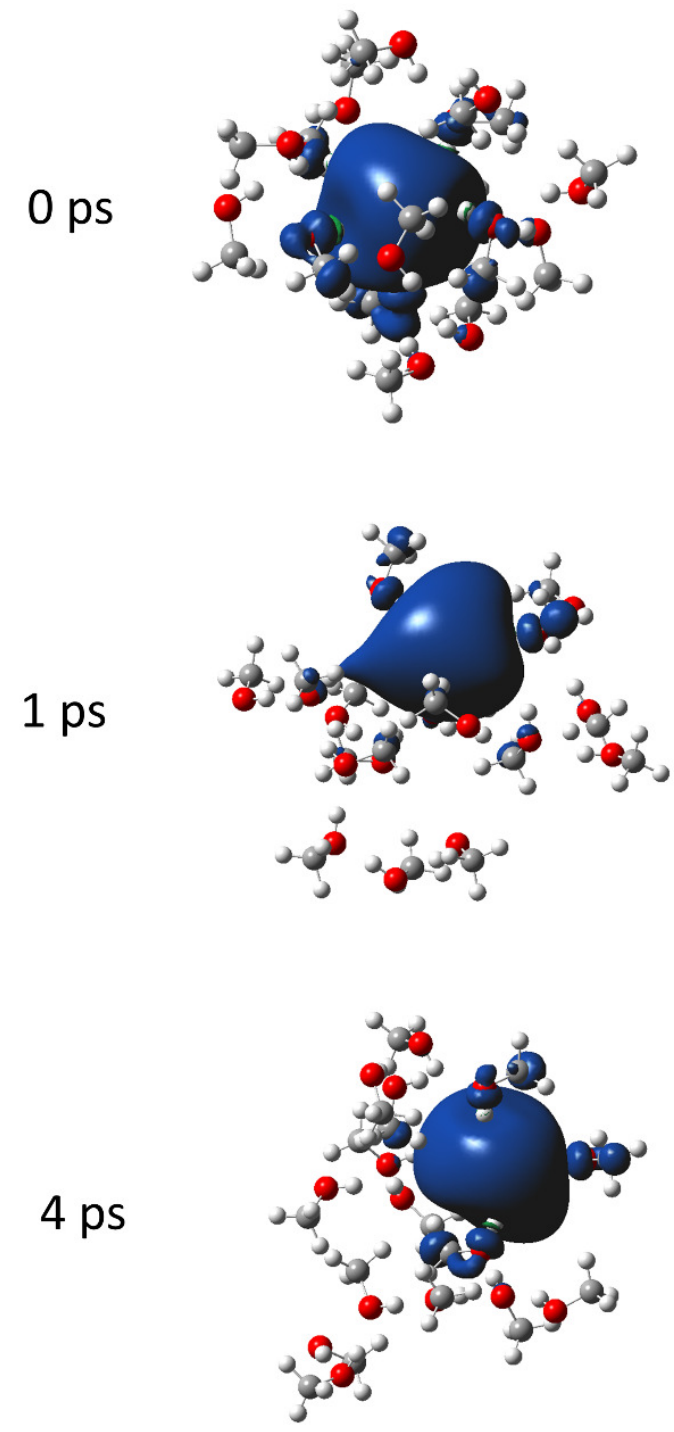

10 ps

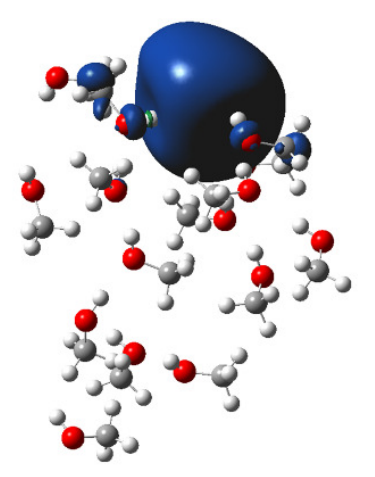


Figure 4.

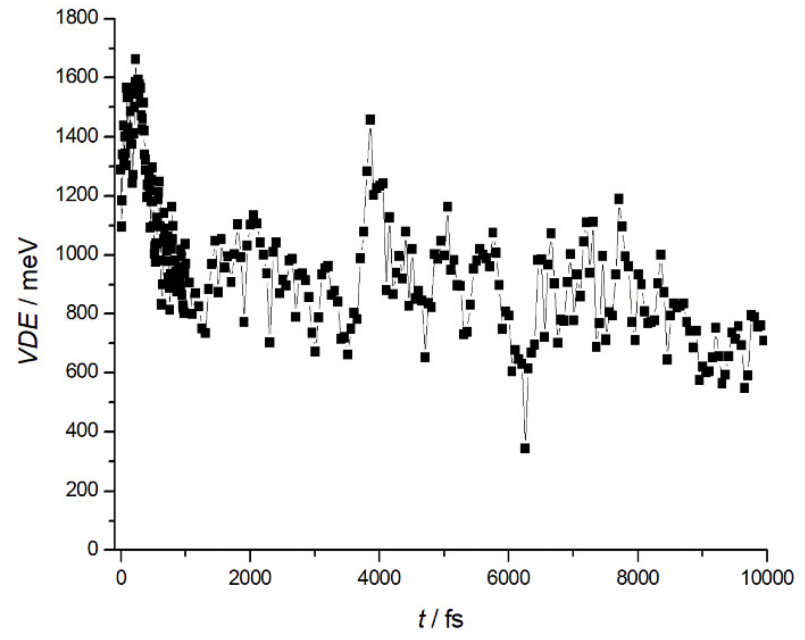


Figure 5.

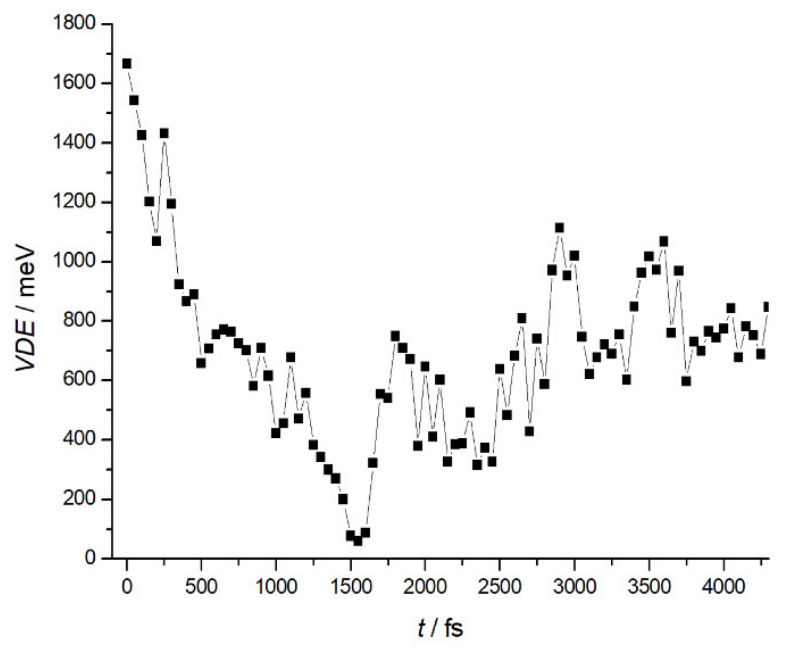


Figure 6.
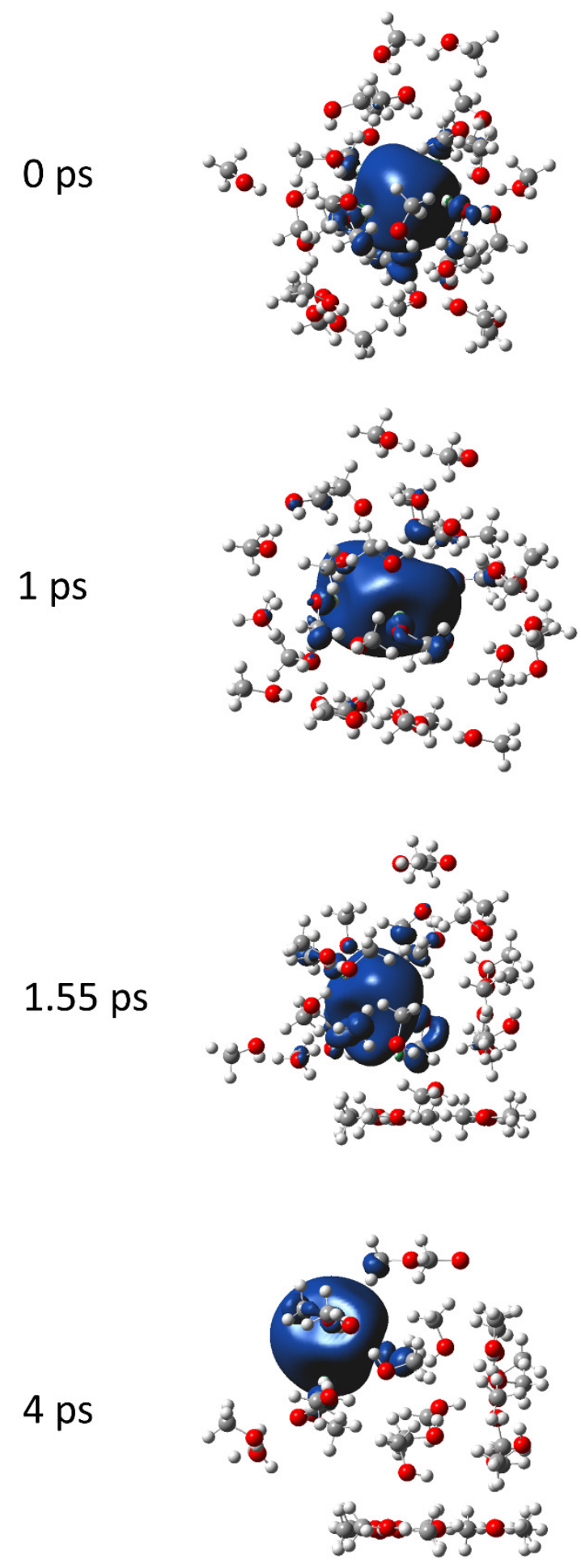
Figure 7.

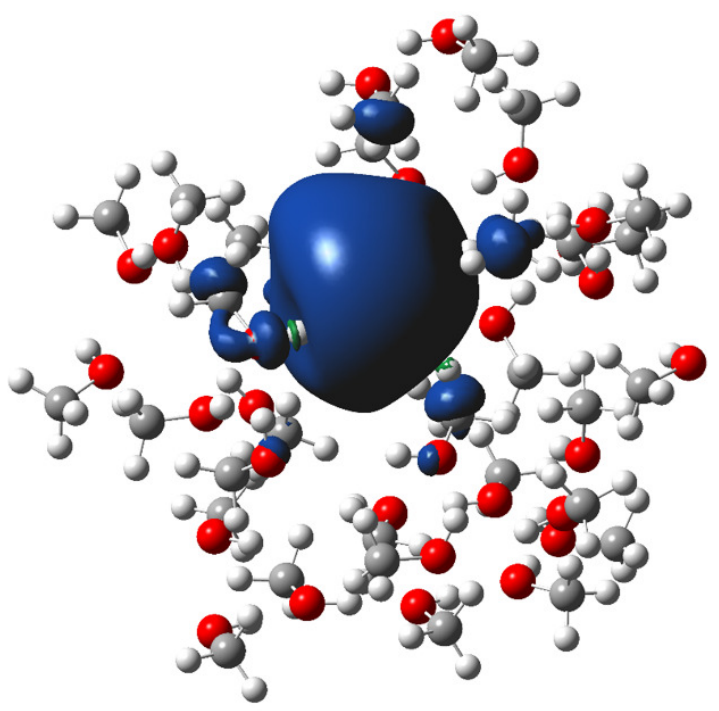


Figure 8.
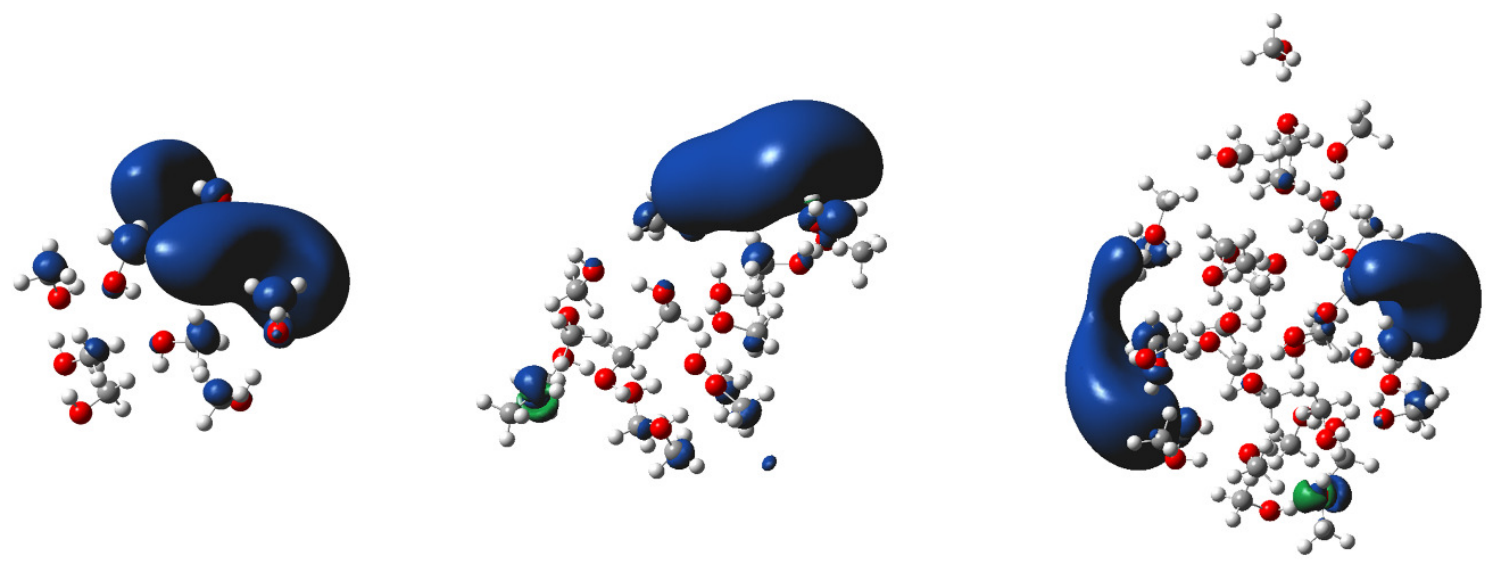

$\left(\mathrm{CH}_{3} \mathrm{OH}\right)_{8}^{-}$

$\left(\mathrm{CH}_{3} \mathrm{OH}\right)_{16}^{-}$

$\left(\mathrm{CH}_{3} \mathrm{OH}\right)_{32}^{-}$ 
Figure 9.
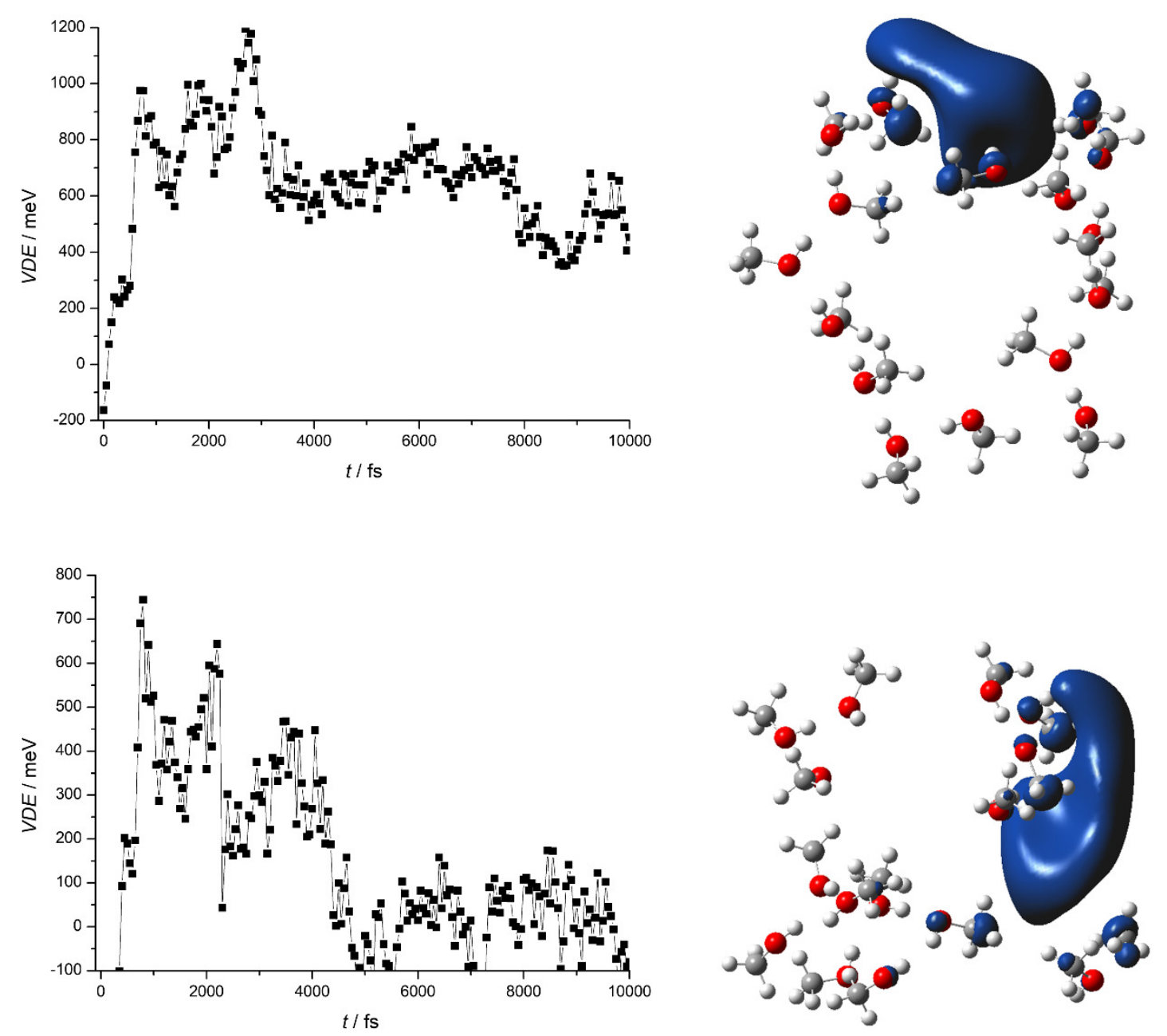


\section{Table of Contents Entry}

Stable surface excess electronic states in small methanol cluster anions were identified and characterized in ab initio molecular dynamics simulations.

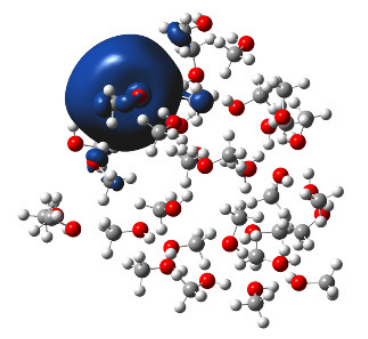




\section{References}

${ }^{1}$ B. C. Garrett, D. A. Dixon, D. M. Camaioni, D. M. Chipman, M. A. Johnson, C. D. Jonah, G. A. Kimmel, J. H. Miller, T. N. Rescigno, P. J. Rossky, S. S. Xantheas, S. D. Colson, A. H. Laufer, D. Ray, P. F. Barbara, D. M. Bartels, K. H. Becker, K. H. Bowen, Jr., S. E. Bradforth, I. Carmichael, J. V. Coe, L. R. Corrales, J. P. Cowin, M. Dupuis, K. B. Eisenthal, J. A. Franz, M. S. Gutowski, K. D. Jordan, B. D. Kay, J. A. LaVerne, S. V. Lymar, T. E. Madey, C. W. McCurdy, D. Meisel, S. Mukamel, A. R. Nilsson, T. M. Orlando, N. G. Petrik, S. M.

Pimblott, J. R. Rustad, G. K. Schenter, S. J. Singer, A. Tokmakoff, L.-S. Wang, C. Wittig and T. S. Zwier, Chem. Rev., 2005, 105, 355-390.

2 J. H. Baxendale and P. Wardman, J. Chem. Soc., Faraday Trans. 1, 1973, 69, 584.

${ }^{3}$ a) D. F. Feng and L. Kevan, Chem. Rev., 1980, 80, 1-20; b) L. Kevan, Radiat. Phys. Chem., 1981, 17, 413-423; c) L. Kevan, Chem. Phys. Lett., 1979, 66, 578-580.

${ }^{4}$ F. Y. Jou and G. R. Freeman, Can. J. Chem., 1979, 57, 591-597; F. Y. Jou and G. R. Freeman, J. Phys. Chem., 1977, 81, 909-915.

${ }^{5}$ X. Shi, F. H. Long, H. Lu and K. B. Eisenthal, J. Phys. Chem., 1995, 99, 6917-6922.

${ }^{6}$ P. K. Walhout, J. C. Alfano, Y. Kimura, C. Silva, P. Reid and P. F. Barbara, Chem. Phys. Lett., 1995, 232, 135-140.

${ }^{7}$ C. M. Stuart, M. J. Tauber and R. A Mathies, J. Phys. Chem. A, 2007, 111, 8390-8400.

${ }^{8}$ H. Shen, N. Kurahashi, T. Horio, K. Sekiguchi and T. Suzuki, Chem. Lett., 2010, 39, 668670.

${ }^{9}$ T. Horio, H. Shen, S. Adachi and T. Suzuki, Chem. Phys. Lett., 2012, 535, 12-16.

${ }^{10}$ A. Kammrath, J. R. R. Verlet, G. B. Griffin and D. M. Neumark, J. Chem. Phys., 2006, 125, 171102.

${ }^{11}$ A. Kammrath, G. B. Griffin, J. R. R. Verlet, R. M. Young and D. M. Neumark, J. Chem. Phys., 2007, 126, 244306. 
${ }^{12}$ M. H. Elkins, H. L. Williams and D. M. Neumark, J. Chem. Phys., 2015, 142, 234501.

${ }^{13}$ R. M. Young and D. M. Neumark, Chem. Rev., 2012, 112, 5553-5577.

${ }^{14}$ D. H. Paik, I-R. Lee, D.-S. Yang, J. S. Baskin and A. H. Zewail, Science, 2004, 306, 672675.

${ }^{15}$ J. R. R. Verlet, A. E. Bragg, A. Kammrath, O. Cheshnovsky and D. M. Neumark, Science, 2005, 307, 93-96.

${ }^{16}$ L. Ma, K. Majer, F. Chirot and B. v. Issendorff, J. Chem. Phys., 2009, 131, 144303.

${ }^{17}$ F. Zappa, S. Denifl, I. Mähr, A. Bacher, O. Echt, T. D. Märk and P. Scheier, J. Am. Chem. Soc., 2008, 130, 5573-5578.

${ }^{18}$ G. B. Griffin, R. M. Young, O. T. Ehrler and D. M. Neumark, J. Chem. Phys., 2009, 131, 194302.

${ }^{19}$ O. T. Ehrler and D. M. Neumark, Acc. Chem. Res., 2009, 42, 769-777.

${ }^{20}$ R. N. Barnett, U. Landman, C. L. Cleveland and J. Jortner, Phys. Rev. Lett., 1987, 59, 811814.

${ }^{21}$ L. Turi, W.-S. Sheu and P. J. Rossky, Science, 2005, 309, 914-917.

${ }^{22}$ L. D. Jacobson and J. M. Herbert, J. Am. Chem. Soc., 2011, 133, 19889-19899.

${ }^{23}$ R. N. Barnett, R. Giniger, O. Cheshnovsky and U. Landman, J. Phys. Chem. A., 2011, 115, 7378-7391.

${ }^{24}$ J. Zhu and R. I. Cukier, J. Chem. Phys., 1993, 98, 5679-5693.

${ }^{25}$ L. Turi, A. Mosyak and P. J. Rossky, J. Chem. Phys., 1997, 107, 1970-1980.

${ }^{26}$ A. Mosyak, P. J. Rossky and L. Turi, Chem. Phys. Lett., 1998, 282, 239-244.

${ }^{27}$ A. Mosyak, O. V. Prezhdo and P. J. Rossky, J. Mol. Struct., 1999, 485-486, 545-554.

${ }^{28}$ P. Mináry, L. Turi and P. J. Rossky, J. Chem. Phys., 1999, 110, 10953-10962.

${ }^{29}$ L. Turi, P. Mináry and P. J. Rossky, Chem. Phys. Lett., 2000, 316, 465-470.

${ }^{30}$ L. Turi and P. J. Rossky, J. Chem. Phys., 2004, 120, 3688-3698. 
${ }^{31}$ L. Mones and L. Turi, J. Chem. Phys., 2010, 132, 154507.

${ }^{32}$ L. Mones, P. J. Rossky and L. Turi, J. Chem. Phys., 2010, 133, 144510.

${ }^{33}$ L. Turi, Á. Madarász and P. J. Rossky, J. Chem. Phys., 2006, 125, 014308.

${ }^{34}$ U. Buck and F. Huisken, Chem. Rev., 2000, 100, 3863-3890.

${ }^{35}$ L. Mones, P. J. Rossky and L. Turi, J. Chem. Phys., 2011, 135, 084501.

${ }^{36}$ M. Boero, M. Parrinello, K. Terakura, T. Ikeshoji and C. C. Liew, Phys. Rev. Lett., 2003, 90, 226403.

${ }^{37}$ T. Frigato, J. VandeVondele, B. Schmidt, C. Schütte and P. Jungwirth, J. Phys. Chem. A, 2008, 112, 6125-6133.

${ }^{38}$ O. Marsalek, F. Uhlig and P. Jungwirth, J. Phys. Chem. C, 2010, 114, 20489-20495.

${ }^{39}$ O. Marsalek, F. Uhlig, T. Frigato, B. Schmidt and P. Jungwirth, Phys. Rev. Lett., 2010, 105, 043002.

${ }^{40}$ F. Uhlig, O. Marsalek and P. Jungwirth, J. Phys. Chem. Lett., 2012, 3, 3071-3075.

${ }^{41}$ F. Uhlig, O. Marsalek and P. Jungwirth, J. Phys. Chem. Lett., 2013, 4, 338-343.

${ }^{42}$ F. Uhlig, J. M. Herbert, M. P. Coons and P. Jungwirth, J. Phys. Chem. A, 2014, 118, 75077515.

${ }^{43}$ J. P. Perdew, K. Burke and M. Ernzerhof, Phys. Rev. Lett., 1996, 77, 3865-3868.

${ }^{44}$ C. Adamo and V. Barone, J. Chem. Phys., 1999, 110 6158-6170.

${ }^{45}$ A. D. Becke, Phys. Rev. A, 1988, 38, 3098-3100.

${ }^{46}$ C. Lee, W. Yang and R. G. Parr, Phys. Rev. B, 1988, 37, 785-789.

${ }^{47}$ L. Turi, J. Chem. Phys., 2016, 144, 154311.

${ }^{48}$ G. Pohl, L. Mones and L. Turi, J. Chem. Phys., 2016, 145, 164313.

${ }^{49}$ H. Iikura, T. Tsuneda, T. Yanai and K. Hirao, J. Chem. Phys., 2001, 115, 3540-3544.

${ }^{50}$ A. D. Becke, J. Chem. Phys., 1993, 98, 1372-1377. 
${ }^{51}$ J. C. Rienstra-Kiracofe, G. S. Tschumper, H. F. Schaefer, S. Nandi and G. B. Ellison, Chem. Rev., 2002, 102, 231-282.

${ }^{52}$ R. E. Larsen, W. J. Glover and B. J. Schwartz, Science, 2010, 329, 65-69.

${ }^{53}$ J. VandeVondele, M. Krack, F. Mohamed, M. Parrinello, T. Chassaing and J. Hutter, Comput. Phys. Commun., 2005, 167, 103-128.

${ }^{54}$ S. Goedecker, M. Teter and J. Hutter, Phys. Rev. B, 1996, 54, 1703-1710.

${ }^{55}$ C. Hartwigsen, S. Goedecker and J. Hutter, Phys. Rev. B, 1998, 58, 3641-3662.

${ }^{56}$ J. VandeVondele and J. Hutter, J. Chem. Phys., 2007, 127, 114105.

${ }^{57}$ W. Hehre, R. Ditchfield and J. A. Pople, J. Chem. Phys., 1972, 56, 2257-2261; M. J. Frisch, J. A. Pople and J. S. Binkley, J. Chem. Phys., 1984, 80, 3265-3269.

${ }^{58}$ J. M. Herbert and M. Head-Gordon, J. Phys. Chem. A, 2005, 109, 5217-5229.

${ }^{59}$ M. Guidon, J. Hutter and J. J. VandeVondele, J. Chem. Theory Comput., 2010, 6, 23482364.

${ }^{60}$ G. J. Martyna and M. E. Tuckerman, J. Chem. Phys., 1999, 110, 2810-2821.

${ }^{61}$ G. Bussi, D. Donadio and M. Parrinello, J. Chem. Phys., 2007, 126, 014101.

${ }^{62}$ Gaussian 09, Revision B.01, M. J. Frisch, G. W. Trucks, H. B. Schlegel, G. E. Scuseria, M. A. Robb, J. R. Cheeseman, G. Scalmani, V. Barone, B. Mennucci, G. A. Petersson, H. Nakatsuji, M. Caricato, X. Li, H. P. Hratchian, A. F. Izmaylov, J. Bloino, G. Zheng, J. L. Sonnenberg, M. Hada, M. Ehara, K. Toyota, R. Fukuda, J. Hasegawa, M. Ishida, T. Nakajima, Y. Honda, O. Kitao, H. Nakai, T. Vreven, J. A. Montgomery, Jr., J. E. Peralta, F. Ogliaro, M. Bearpark, J. J. Heyd, E. Brothers, K. N. Kudin, V. N. Staroverov, R. Kobayashi, J. Normand, K. Raghavachari, A. Rendell, J. C. Burant, S. S. Iyengar, J. Tomasi, M. Cossi, N. Rega, J. M. Millam, M. Klene, J. E. Knox, J. B. Cross, V. Bakken, C. Adamo, J. Jaramillo, R. Gomperts, R. E. Stratmann, O. Yazyev, A. J. Austin, R. Cammi, C. Pomelli, J. W. Ochterski, R. L. Martin, K. Morokuma, V. G. Zakrzewski, G. A. Voth, P. Salvador, J. J. Dannenberg, S. Dapprich, A. 
D. Daniels, Ö. Farkas, J. B. Foresman, J. V. Ortiz, J. Cioslowski, and D. J. Fox, Gaussian, Inc., Wallingford CT, 2009.

${ }^{63}$ L. Turi, J. Chem. Phys., 1999, 110, 10364-10369.

${ }^{64}$ J. A. Walker and D. M. Bartels J. Phys. Chem. A, 2016, 120, 7240-7247.

${ }^{65}$ J. Liu, R. I. Cukier, Y. Bu and Y. Shang J. Chem. Theory Comput., 2014, 10, 4189-4197. 